Service social

\title{
Une conception alternative de la violence conjugale : vers une intervention efficace auprès des hommes violents
}

\section{Jürgen Dankwort}

Volume 37, numéro 1-2, 1988

Par-delà les barrières des sexes

URI : https://id.erudit.org/iderudit/706387ar

DOI : https://doi.org/10.7202/706387ar

Aller au sommaire du numéro

Éditeur(s)

École de service social de l'Université Laval

ISSN

1708-1734 (numérique)

Découvrir la revue

Citer cet article

Dankwort, J. (1988). Une conception alternative de la violence conjugale : vers une intervention efficace auprès des hommes violents. Service social, 37(1-2), 86-119. https://doi.org/10.7202/706387ar
Résumé de l'article

Face à ce débat inextricable sur la nature de la violence conjugale, et devant la controverse que soulève la mise en place d'organisations destinées aux hommes dans ce nouveau secteur de préoccupations sociales, l'auteur relève quatre tendances générales qui ont influencé les ressources, programmes et stratégies d'intervention conçus pour les hommes violents. Il examine différentes théories psychothérapeutiques qui, combinées aux théories sur l'apprentissage social et enrichies des perspectives socioculturelles, mènent vers une conception féministe de la violence conjugale et une intervention proféministe auprès des hommes violents. 


\section{Une conception alternative de la violence conjugale : vers une intervention efficace auprès des hommes violents}

\section{Jürgen Dankwort}

En 1985, il n'existait dans tout le Québec que trois programmes destinés aux hommes violents. Depuis, plus d'une douzaine de projets ont vu le jour dans l'ensemble de la province, sous différents auspices allant des organisations communautaires au réseau des Centres locaux de services communautaires (C.L.S.C.) financés par le gouvernement. Ici comme dans le reste de l'Amérique du Nord, ces programmes d'intervention ont été mis en place une fois que les mouvements pour la défense des femmes battues sont parvenus à faire reconnaître la violence conjugale comme un véritable problème social qui opprime près d'un million de Canadiennes, et non plus comme de simples "chicanes de ménage" (MacLeod, 1987: 7).

Sans l'intervention des femmes qui, à force de pressions, ont réussi à inscrire la violence conjugale à l'ordre du jour, les programmes destinés aux hommes violents ne se développeraient sans doute pas à l'allure que l'on connaît actuellement dans la province. II n'en demeure pas moins que ces programmes ont généralement été mis sur pied, au Québec, par des organisations indépendantes des mouvements pour les femmes victimes de violence. De plus, alors qu'on peut retracer I'histoire et l'idéologie du mouvement des femmes et des services destinés aux Québécoises victimes de violence (Beaudry, 1984 ; Carrier et Michaud, 1982), les origines et les fondements idéologiques des programmes d'intervention auprès des hommes sont assez mal connus.

Par ailleurs, contrairement à une opinion largement répandue, les principes idéologiques qui sous-tendent plusieurs programmes destinés aux hommes sont issus de considérations sur la «libération de l'homme " 
et la « condition masculine » nettement en désaccord avec la plupart des idées fondamentales avancées par les mouvements féministes (Carrigan et al., 1987 ; Dulac, 1984). Bien qu'il y ait consensus sur le fait que les hommes doivent cesser de minimiser ou de nier leur comportement violent pour devenir des individus responsables et des partenaires non dangereux, on ne s'accorde pas sur la cause ou les causes de la violence masculine, ni sur le rôle de l'homme comme instigateur de la violence et sa réticence à accepter la responsabilité de son comportement violent.

Dans la conjoncture présente, nous avons conscience des tensions qui existent dans la province entre le réseau des maisons d'hébergement pour femmes battues et les programmes pour hommes violents. Deux récents numéros du bulletin de liaison du Regroupement provincial des maisons d'hébergement et de transition pour femmes victimes de violence, Avalanche, ont porté un regard critique sur la prolifération des programmes pour hommes violents au Québec (vol. 3, n 9), et analysé la manière dont les théories actuelles sur la violence conjugale camouflent les vraies causes de cette violence et nous détournent d'une intervention responsable et soucieuse de la sécurité de la femme (vol. 4, $n^{\circ} 12$ ). (Un extrait de la version préliminaire de cet article a été publié dans le numéro de février 1989 de ce bulletin.) D'ailleurs, une étude descriptive des programmes québécois pour hommes violents, commandée par le Ministère de la santé et des services sociaux, confirme la présence de théories concurrentes sur la violence masculine. De plus, cette diversité se traduit dans différentes stratégies d'intervention dont la plupart n'ont pas reconnu la pertinence d'une orientation féministe dans le traitement des agresseurs (Rondeau et al., 1988).

Le développement de programmes pour hommes violents a soulevé des tensions semblables dans les autres provinces et pays où ils ont été mis en place. Par exemple, la création de tels programmes a suscité des critiques en Ontario car les subventions attribuées pour leur implantation ont été prises sur les fonds alloués aux services pour femmes battues (McEvoy, 1984). En Angleterre, comme dans d'autres pays d'outre-mer, les instances gouvernementales et/ou les organisations communautaires envisagent actuellement l'instauration de tels programmes même si, en règle générale, l'arrestation pour violence conjugale est encore peu répandue et les services pour les victimes, souvent inadéquats. Là où des programmes pour hommes violents sont en cours d'élaboration ou déjà en place, leur efficacité, la répartition des ressources et du financement pour réhabiliter les participants de même que les théories contradictoires sur la violence font l'objet de controverses tout à fait récentes (Cuffe, 1989). 
Par contre, aux États-Unis, un bon nombre de ces programmes, réalisés en collaboration avec la National Coalition Against Domestic Violence, ont dressé une liste de recommandations pour s'assurer que l'intervention auprès des hommes violents se fasse de manière responsable et non dangereuse, sous supervision du mouvement des femmes battues. On évite ainsi toute rivalité dans l'attribution des subventions. Cet état d'esprit a également permis aux diverses organisations d'hommes et de femmes d'arriver à des ententes importantes sur la nature et les méthodes d'intervention auprès des hommes violents et sur la nécessité de changements sociaux-juridiques (National Coalition Against Domestic Violence, 1982).

Face à ce débat inextricable sur la nature de la violence conjugale, et devant la controverse que soulève la mise en place d'organisations destinées aux hommes dans ce nouveau secteur de préoccupations sociales, nous avons relevé quatre tendances générales qui ont influencé les programmes conçus pour les hommes qui battent leur femme. Sans s'exclure mutuellement, ces diverses tendances débouchent sur des conclusions spécifiques, parfois contradictoires, quant à savoir qui est foncièrement responsable de la violence et qui en est vraiment victime. Les réponses à ces questions vont grandement influencer le choix des ressources et des stratégies d'intervention, les plus appropriées, pour mettre un terme à la violence conjugale.

Tenant compte de ces considérations et largement inspiré par des textes de Okun (1986), Adams (1988) et Breines \& Gordon (1983), nous examinerons 1 ) différentes théories psychothérapeutiques qui, combinées aux 2) théories sur l'apprentissage social et enrichies par des 3) perspectives socio-culturelles, nous ont mené vers une 4) conception féministe de la violence conjugale et une intervention proféministe auprès des hommes violents.

\section{Le point de vue psychothérapeutique}

\section{La relation entre la colère et la violence}

La façon traditionnelle d'envisager la relation entre la colère et la violence repose essentiellement sur les écrits de Freud et de Darwin. Selon eux, il existe un lien organique entre la colère et la violence. Les hommes sont agressifs parce que la nature en a décidé ainsi afin d'assurer la reproduction de l'espèce. C'est donc un comportement " instinctif " ou " naturel " que de soumettre l'objet sexuel ou de vaincre sa résistance (Freud, 1938: 569). L'agressivité a presque toujours été 
mise en relation avec les "instincts" ou les "pulsions» biologiques, jusqu'à ce que des théoriciens plus contemporains viennent remettre en question cette vision déterministe de la violence des hommes envers leur partenaire.

Cependant, de nombreux spécialistes persistent à affirmer que, pour des raisons d'ordre biologique, les hommes sont davantage prédisposés à la violence que les femmes. En dépit de l'absence de preuves expérimentales concernant les êtres humains, une théorie populaire se perpétue selon laquelle la testostérone, hormone sexuelle mâle, fait de la violence une attitude " intrinsèque ", " fondamentale " et " naturelle" chez les garçons et chez les hommes (Konner, 1988). Selon cette opinion émise sur les particularités du métabolisme masculin, les hommes sont plus enclins à la violence. Ils apparaissent ainsi, dans une large mesure, comme des victimes à la merci de leurs caractéristiques génétiques ou biologiques. Toute stratégie d'intervention devrait donc tenir compte de cette situation et s'efforcer de leur fournir les moyens de surmonter ou de contrôler ces tendances violentes; elle hésitera donc à leur reprocher une conduite qui, de toute évidence, échappe partiellement à leur contrôle.

\section{La violence en tant que psychopathologie}

Dans les cas où psychiatres et psychothérapeutes identifient la violence comme un problème dans les relations interpersonnelles, ils la considèrent généralement comme le produit de facteurs intra-psychiques qui, s'ils n'étaient pas corrigés par une "introspection ", aboutiraient à des comportements violents. Dans les rapports cliniques, on décrit les personnes violentes comme ayant des personnalités de type " obsessivocompulsif ", " paranoïaque " ou, à la limite, comme des malades capables "d'actes agressifs irrationnels» (Shainess, $1977:$ 114-155). Faulk (1977), par exemple, parle de "l'anormalité psychiatrique" et de l'état de déséquilibre mental de ces patients $(1977: 121,124)$. Snell, Rosenwald et Robey (1964) ont construit une théorie psychanalytique de la violence familiale à partie du concept freudien du masochisme féminin selon lequel la femme entretient la violence conjugale et le comportement violent du mari, satisfait ses besoins, et instaure ainsi un certain «équilibre " au sein du couple. Ainsi, les intenses besoins de dépendance du mari satisfont les besoins de la femme dans leur relation de symbiose. La violence représentait pour le mari une manière de réaffirmer sa masculinité par rapport à sa désaffection d'homme "timide». La punition et l'humiliation étaient supposées fournir à la femme une "gratification 
masochiste » étant donné la culpabilité qu'elle avait accumulée à cause de son " comportement castrateur et contrôlant» (1964: 110-111).

\section{Les femmes trompeuses et masochistes}

C'est dans le cadre de la psychopathologie que sont nés les doutes à propos de ce que rapportaient les femmes qui se disaient victimes de violence. Mackinnon (1987: 144) nous rappelle que Freud avait pris pour parti de ne pas croire les femmes qui disaient avoir été l'objet d'abus sexuels pendant leur enfance. Freud est en effet revenu sur ses premières déclarations à propos de la violence faite aux femmes dans la société victorienne, en affirmant que les symptômes hystériques observés chez ses patientes relevaient plutôt de fantasmes sexuels que d'une violence vraiment vécue. Cette attitude qui consiste à sous-estimer ou même à nier ce type de violence prévaut encore aujourd'hui. Elle a pour effet de négliger le contexte social à l'intérieur duquel les troubles psychologiques se manifestent (Rieker et Carmen, 1986).

Okun (1986), citant plusieurs auteurs, a démontré comment les travaux de Freud et de Deutsch ont contribué à faire du masochisme un trait proprement féminin et un thème populaire auprès des thérapeutes.

"On peut toujours présumer que la victime a joué un rôle crucial lors de l'offense et qu'elle a pu, directement ou indirectement, provoquer ou précipiter la violence qu'elle a subie. »(Schultz, 1960 : 103.)

La thèse de cette auteure est acceptable dans la mesure où elle emploie le terme " masochiste » dans le sens qu'on lui reconnaît aujourd'hui. Masochisme ne signifie plus prendre plaisir à la souffrance. Cette notion, désormais dépassée, a été remplacée par une théorie plus moderne selon laquelle une piètre estime d'elles-mêmes amène les femmes à se sentir perdantes; cette perception empêcherait les femmes battues d'entreprendre les démarches nécessaires pour mettre fin à une relation où règne la violence.

Les fructueux travaux des Dobash (1979) sur la violence conjugale nous fournissent d'autres exemples qui démontrent bien comment la psychopathologie a influencé notre perception. Les deux auteurs critiquent d'ailleurs ce point de vue, le considérant à la fois sexiste et nuisible aux femmes:

"Une femme ne pourra alors être pleinement féminine que si elle se laisse dominer. La femme provocatrice et agressive appelle souvent, de la sorte inconsciemment, ce qu'elle redoute le plus. En irritant un homme, c'est une réaction de domination qu'elle essaie de susciter... " (Storr, 1974 : 95.) 


\section{Portrait psychologique}

Pour de nombreux thérapeutes contemporains, la personne violente est aux prises avec un ego perturbé, une piètre estime de soi et des besoins narcissiques (tels qu'une surdépendance émotive). Cette situation explique pourquoi elle réagit si fortement aux menaces réelles ou imaginées. Un tel comportement, affirme-t-on, tourne souvent à l'agression car, dans ce processus, un individu diminue l'autre en ayant recours à la violence. On peut relier un tel comportement à des troubles survenus au cours de périodes antérieures du développement, par exemple, un rejet de la part d'un des parents, la surdépendance visà-vis la mère, la crainte du père, etc.

Cependant, si on s'en remet trop aux explications psychiatriques ou psychothérapeutiques, on risque d'oublier le rôle de l'agresseur dans la situation violente et les contraintes qui amènent la victime à ne pas rompre la relation malgré les abus dont elle est l'objet. On laisse entendre que toute femme battue peut mettre fin à la relation si elle le veut. Dans les faits cependant, plusieurs n'en prennent pas l'initiative par crainte pour leur vie ou pour la sécurité financière de la famille. De plus, la violence d'un homme ne s'arrête pas nécessairement au moment où sa femme le quitte. Dans certains cas, elle peut au contraire s'intensifier (Okun, 1986 : 81). Enfin, malgré la croyance populaire, une femme ne s'engage pas toujours dans le mariage ou la cohabitation par choix (Pagelow, 1981). Elle fait des choix à l'intérieur de certaines limites. Un compagnon lui offre une protection contre les étrangers qui peuvent la harceler, voire l'attaquer; de plus, une relation avec un homme lui assure d'autres avantages sociaux et financiers importants dans une société où les femmes (et en particulier les mères célibataires) font l'objet d'une discrimination sociale et économique.

\section{Les implications pratiques : le counseling conjugal}

Cette vision étroite de la violence conjugale sert toutefois aux thérapeutes qui tentent de venir en aide à un nombre toujours croissant de couples qui vivent des conflits. En concentrant leur action sur les problèmes psychologiques de chacun des partenaires ou sur les problèmes de la relation, les thérapeutes croient adopter une position neutre, et ainsi assurer l'efficacité de la thérapie. On a tendance à oublier, dans ce contexte, que le refus du thérapeute de prendre parti et la présence de la victime aux séances de thérapie laissent supposer qu'elle est, d'une certaine manière, elle aussi responsable de la violence. L'homme reçoit un message tout aussi ambigu quant à savoir qui est responsable de la 
violence. S'il est entièrement responsable de la situation, pourquoi sa femme est-elle en thérapie avec lui ? De plus, les femmes placées dans un contexte où elles sont appelées à parler devant leur partenaire de ce qu'elles ont à lui reprocher ou d'une éventuelle rupture, risquent de voir s'intensifier la violence à leur égard.

\section{L'optique psychologique retenue dans les programmes destinés aux hommes}

Dans les récents programmes canadiens spécialement mis sur pied pour traiter la violence conjugale par l'entremise de thérapies de groupe, on accorde beaucoup d'importance à la psychopathologie (Dankwort, 1988[b]). Même les programmes comportant une mise en garde contre la trop grande importance accordée aux facteurs intrapsychiques dans l'analyse de la violence conjugale continuent à définir leur fondement et leur intervention à partir de la " différence " entre les hommes qui battent leur femme et les autres (Currie, 1988; Larouche, 1987 ; Sinclair, 1986). Les tests et les portraits psychologiques, au lieu d'examiner ce que les hommes violents ont en commun avec la population masculine en général, font plutôt ressortir le caractère " anormal » des agresseurs. On parle certes de l'importance de faire en sorte que les hommes acceptent la pleine responsabilité de leurs actes et cessent de rejeter la faute sur leurs partenaires; toutefois, l'histoire psychologique de l'agresseur, son enfance difficile par exemple, est souvent citée comme la cause première de sa violence actuelle (Philibert, 1988).

Quant à la victime, c'est aussi à travers sa psychologie personnelle qu'on cherche à expliquer le fait qu'elle ne quitte pas son partenaire violent, et on laisse de côté les problèmes de contrôle et de domination qui affectent la relation, ou bien on les envisage uniquement dans le contexte psychologique traditionnel. En d'autres mots, la femme battue le serait parce qu'elle est, d'une certaine manière, différente des autres femmes. De plus, l'analyse des rapports de contrôle et de pouvoir démontre ici qu'ils sont manipulés et exploités par deux partenaires égaux. Il est intéressant de constater que des analyses semblables portant sur la personnalité psychologique des prisonniers de camps de concentration ou des réfugiés politiques maltraités, n'ont jamais été diffusées, bien que ces individus partagent plusieurs traumatismes avec les femmes battues : la peur, la réclusion, l'isolement social, les mauvais traitements, les carences de sommeil, les menaces de mort ou de torture, les actes violents imprévisibles, I'humiliation, les dénonciations et l'inculcation de leur propre culpabilité, etc. Okun, parlant du contrôle coercitif dont les femmes battues sont l'objet, pose la question suivante : 
pourquoi soupçonne-t-on ces femmes, qui se trouvent dans une situation presque identique à celle des prisonniers qui subissent des lavages de cerveau, d'être des provocatrices et des masochistes ? Il serait choquant de lire de longues études sur les éventuelles caractéristiques susceptibles d'avoir prédisposé les survivants des camps de concentration à être des victimes. Dès qu'on admet le rôle central de la dynamique du contrôle coercitif dans la violence faite aux femmes, on évite d'aboutir à des conclusions qui font porter la responsabilité de la violence sur la victime ou présentent cette dernière comme encourageant les mauvais traitements qu'elle subit (Okun, 1986 : 88-89). Cette façon de voir va totalement à l'encontre de ce qu'on entend ou lit habituellement (Norwood, 1987), à savoir que les femmes s'embarquent et demeurent dans des relations violentes parce qu'elles ont fait de mauvais choix et, par la suite, deviennent incapables de rompre les liens affectifs qui les attachent à leur partenaire.

\section{Les théories de l’apprentissage social}

\section{Le legs du déterminisme}

Avec le temps, on a remis en cause le déterminisme de la pensée de Freud et de Darwin en ce qui a trait à la violence. Les critiques font ressortir qu'aux facteurs biologiques s'ajoutent la nature de la provocation et les circonstances contraignantes particulières qui peuvent aussi influencer la réaction de l'individu. Bandura (1973) et Novaco (1975) affirment qu'il est possible d'éprouver de la colère sans nécessairement poser des gestes violents et que, inversement, un individu peut être amené à poser de tels gestes sans ressentir de colère. Toch (1969) récuse aussi cette façon de voir la violence comme un simple réflexe de colère. Il fait remarquer que le comportement violent a toujours un but précis, qu'il est prévisible et répétitif.

\section{Le behaviorisme et les théories des rôles des sexes}

Le behaviorisme nous a démontré comment nous avons appris certains comportements par l'entremise de conditionnements, sociaux notamment. Plusieurs intervenants dans le domaine de la violence envers les femmes en ont fait le thème central de leurs travaux (Ganley et Harris, 1978 ; Saunders, 1977 ; Walker, 1978). Les adeptes de la théorie 
des rôles des sexes ont, pour leur part, ajouté que la pression sociale exercée sur les garçons et sur les hommes afin qu'ils adoptent le comportement "approprié", fait naître en eux certaines craintes et tensions qui proviennent du rejet des aspects féminins de leur personnalité ainsi que de tous les désavantages associés à la condition masculine (Goldberg, 1982; Mead, 1972; Steinmann et Fox, 1974). Si l'on regarde les choses sous cet angle, on ne peut plus considérer les hommes violents comme des malades mentaux réagissant à des instincts biologiques. On les voit plutôt comme des hommes normaux souffrant d'une piètre estime d'eux-mêmes, éventuellement coincés dans une identité sexuelle imposée par la société, et susceptibles de réagir agressivement au stress à cause, justement, du comportement qu'ils ont appris. II faudrait qu'ils se "libèrent» et deviennent plus androgynes, moins aliénés et davantage à l'écoute de leurs sentiments. La solution aux troubles du comportement masculin passe par la « libération de l'homme " (Farrel, 1974; Nichols, 1975 ; Naifeh et Smith, 1987).

D'une part, la théorie de l'apprentissage souligne la nécessité d'un renforcement négatif du comportement violent pour que celui-ci cesse (Okun, 1986: 83). L'application de ce principe dans le cadre d'une intervention suppose que l'homme violent soit tout de même tenu responsable de son comportement qui constitue une agression criminelle. Par contre, l'argument selon lequel l'agressivité de l'homme violent risque d'augmenter face à la frustration peut avoir pour conséquence de détourner ce dernier de l'idée qu'il est entièrement responsable de ses actes. Par ailleurs, on peut mettre en doute l'hypothèse selon laquelle la frustration entraîne l'agression, car elle ne parvient pas à expliquer pourquoi bien des hommes stressés et frustrés ne battent pas leur femme, ni pourquoi la violence conjugale est également très répandue dans les familles de niveau socio-économique élevé, où les tensions et le stress devraient être moindres.

\section{Des explications contradictoires}

Ces nouvelles considérations sur "l'agression acquise » et les tensions associées au rôle que la société impose aux hommes aboutissent à des explications contradictoires et à des recommandations divergentes quant à savoir qui est responsable de la violence conjugale et quels en sont les remèdes possibles. Geller (1982), dans le cadre d'une approche systémique de la famille, affirme que l'homme qui bat sa femme est le seul responsable de la violence. Pourtant, elle parle "d'impulsions agressives », "d'impulsions violentes » $(1982: 202)$ et « d'agression incontrôlable» (1982: 200). Elle conseille donc fortement aux femmes victimes 
de violence d'agir prudemment avec leur mari afin d'éviter de nouvelles agressions (1982: 203). Des expressions telles que "éclats d'humeur", "éruptions de violence " et " rage explosive " démontrent bien l'enracinement de cette idée selon laquelle la violence correspond à une manifestation d'origine organique ou psychogène. Les travaux actuels sur le sujet, et les interventions qui en résultent, font ressortir que théoriciens et praticiens, malgré leurs perspectives différentes, partagent néanmoins l'idée selon laquelle les hommes violents "perdent le contrôle" de leurs actes (Walker, 1979; Deschner, 1984 ; Guèvremont, 1986 ; Larouche, 1987). On insiste tant sur cette impulsivité mal définie, ou encore cette réaction conditionnée face au stress - toutes deux si difficiles à corriger - qu'on en vient parfois à considérer que l'homme violent ne peut tout simplement pas faire autrement.

\section{Conséquences sur les programmes destinés aux hommes}

Cette façon de considérer les hommes qui battent leur femme a, bien sûr, des conséquences sur les programmes qui leur sont destinés et dont le but est de les aider à mettre fin à leurs comportements violents. Bien que la majorité des Québécois ne soient pas opposés à la politique gouvernementale qui, depuis 1986, prévoit l'arrestation des auteurs de violence conjugale, les intervenants des programmes destinés aux hommes ont déjà émis, quant à eux, des réserves à l'idée de punir ces contrevenants. Ils se demandent d'abord si l'incarcération représente vraiment une mesure appropriée, même dans les cas où il est évident qu'il y a eu violence criminelle. Plusieurs considèrent en effet que la prison constitue elle-même une école de violence et que le placement de ces hommes dans un environnement carcéral ne fera que renforcer leur violence. À leur avis, la thérapie ne peut que donner de meilleurs résultats. Il est intéressant de constater que ce type de réflexion est rare quand il s'agit d'autres crimes impliquant également la violence. Toutefois, le principe selon lequel "la violence entraîne la violence " pourrait tout autant s'appliquer au vol à main armée ou à n'importe quel crime où il est fait usage de la force.

Cette idée selon laquelle la justice criminelle doit traiter la violence conjugale à part, provient, en partie, d'une tendance à "médicaliser » tout comportement qui s'écarte de la norme. L'incarcération apparaît comme une mesure beaucoup trop sévère pour quelqu'un qui a perdu le contrôle de lui-même sous l'effet de l'impulsivité ou de forces qui le dépassent. Quand on évoque l'intervention sociale pour enrayer la violence conjugale, ce même raisonnement refait surface : la motivation 
ou la cause de la violence est extirpée de la volonté pour être relocalisée quelque part dans le corps (problème physiologique) ou dans la tête (problème mental) (Conrad et Schneider, $1980:$ 272-273). Les tentatives récentes visant à appliquer aux hommes violents la théorie de Lenore Walker sur l'impuissance acquise (learned helplessness) démontrent bien à quel point cette façon de voir est répandue (Deschner, 1984 ; Larouche, 1987). Ces hommes, tout comme les femmes violentées, sont considérés comme les " prisonniers » de relations destructives, du fait de leurs expériences passées qui leur ont appris que leur situation ne s'améliorera pas quoi qu'ils ou elles fassent. Cette théorie est toutefois incomplète puisqu'elle ne tient pas compte des influences sociales qui encouragent la victime (ou l'agresseur) à ne pas mettre un terme à la relation violente. De plus, elle ne parvient pas à expliquer, par exemple, pourquoi certaines femmes arrivent à mettre fin à ce type de relation après seulement quelques épisodes violents (Okun, 1986 : 85). Selon Breines et Gordon (1983: 508-509), cette perspective n'exclut pas la psychopathologie, mais la remplace par un modèle psychologique behavioriste qui permet d'expliquer pourquoi certaines personnes sont plus violentes que d'autres.

\section{Les agresseurs impuissants}

Même dans le cadre de programmes destinés aux hommes violents où l'on encourage les clients à accepter leurs sentiments de culpabilité face au comportement répréhensible, l'idée selon laquelle un individu peut perdre ses moyens dans certaines circonstances affectives graves, persiste toujours. Au Canada, tout comme ailleurs en Amérique du Nord, la priorité, dans le cadre des programmes d'intervention, a donc été d'apprendre aux hommes à mieux contrôler leur colère et leur comportement impulsif (Browning, 1984; Dankwort, 1988[a] ; Picard, 1988).

Par exemple, en détachant les notions de pouvoir et d'impuissance de tout contexte socio-politique, et sans tenir compte des particularités liées au genre, Philippe (1984 et 1986) prétend que l'homme bat sa femme " [...] parce qu'il ne peut, ni ne sait comment faire pour expérimenter et assumer l'impuissance quand celle-ci se manifeste ". Selon ce psychologue québécois, plutôt que de rester en contact avec son sentiment de désespoir et d'impuissance, l'homme violent " entraîne sa femme dans son désespoir afin d'alléger une souffrance qui ne peut être que vécue seul». Sa thèse veut que l'homme qui a perdu le contrôle de sa vie le reprenne. Pour le participant qui suit une thérapie de groupe, le défi consiste donc à retrouver son propre pouvoir qui a 
été déplacé, et cela " [...] au prix de reconnaître qu’il est seul à pouvoir faire quelque chose » (1986: 68). Le problème n'est pas en soi l'arrogance qu'un homme manifeste à une femme, mais plutôt la façon dont il s'exprime et agit en utilisant la force physique quand ça va mal en dedans :

\begin{abstract}
"C'est notre expérience à PRO-GAM de constater qu'il n'y a pas beaucoup de différence entre l'homme violent et vous et moi. La seule différence que je connaisse, c'est que nous ne battons pas notre partenaire: contrairement à l'homme qui utilise la violence [parce que] nous avons d'autres alternatives pour exprimer, assumer et agir à partir de la rage qui se manifeste en nous. [...] Comme nous, on ne lui a jamais montré comment être impuissant devant les événements, comment être enragé, comment être triste, comment être heureux..." (1984: 58 et $1986: 68)$.
\end{abstract}

Dès que l'on considère qu'un homme violent agit comme il le fait parce qu'il ne sait pas comment s'y prendre dans des situations stressantes et parce qu'il "perd ses moyens", on considère du même coup qu'il n'est pas entièrement responsable de son comportement. Aussi, certains intervenants croient-ils que l'intervention auprès de ces hommes doit être la moins " menaçante " possible, afin que le client puisse s'ouvrir au changement et guérir de ses traumatismes (Martin, 1988). Ce n'est qu'à cette condition qu'il parviendra à surmonter ses sentiments d'insécurité, à être moins sur la défensive, et à progresser malgré la perception fragile de son identité. Dans la pratique, cela signifie fournir au client un environnement sécurisant et empreint d'empathie dans lequel l'intervenant ne portera aucun jugement et adoptera une attitude de neutralité morale (Buckley et al., 1983 ; Werk et al., 1984 et 1986). Cette conception thérapeutique peut aller jusqu'à exclure que l'on punisse l'auteur d'actes violents ou même qu'on le culpabilise. Une telle démarche irait en effet à l'encontre des objectifs poursuivis.

Bref, dans l'optique des théories de l'apprentissage social, la violence conjugale n'est plus une maladie ni une prédisposition biologique, mais la socialisation d'un comportement ou d'un rôle appris, qui s'accompagne de traces psychologiques de "moments de folie» pour parvenir à combler les lacunes. Le postulat de base, à savoir une causalité psychologique anormale, demeure toutefois inchangé. L'agresseur est encore souvent considéré comme une victime qui mérite aide et compassion. 


\section{La perspective socioculturelle}

\section{Les études sociologiques : du problème personnel à la question sociale}

Après que les mouvements féministes aient dénoncé l'ampleur du problème de la violence conjugale, autour des années 1970 , les sociologues se sont alors penchés plus avant sur la question et, à la suite d'enquêtes scientifiques nationales, en sont arrivés à corroborer ces affirmations (Steinmetz, 1978; Gelles et Steinmetz, 1980; Straus et Gelles, 1986). On a alors assisté à un remaniement de la conception psychopathologique de la violence afin d'incorporer ces nouvelles données, confirmant ainsi le pourcentage élevé de la population aux prises avec ce problème. Ces révélations empiriques ont amoindri la crédibilité de la pathologie individuelle comme facteur dominant dans la violence conjugale. Les chercheurs ont commencé à s'intéresser à l'institution familiale comme foyer de violence.

Selon la perspective socio-culturelle, la violence conjugale est envisagée dans le cadre des inégalités sociales structurelles, des attitudes et des normes culturelles. On a remarqué que les relations conjugales sont particulièrement sensibles au moindre antagonisme, et qu'un contrat de mariage est bien souvent considéré comme un passeport pour la violence. La construction de familles hiérarchiques et nucléaires est de plus propice à l'utilisation de la force dans les relations de supériorité et d'infériorité qui s'y exercent. Pour Straus (1981), il n'est pas plus tolérable de malmener quelqu'un en privé qu'en public, aussi considère-t-il que tous les individus devraient avoir les mêmes droits à l'immunité contre la violence, où qu'ils soient. Cette affirmation est venue appuyer les revendications féministes à l'effet que le mariage ne devienne pas un milieu propice à la réduction des droits civils des femmes ou ne fournisse un cadre permissif à l'intérieur duquel elles peuvent être agressées impunément.

\section{L'influence de la société}

Les recherches anthropologiques de Tavris (1982), entre autres, ont démontré que, dans certaines sociétés, on n'a pas l'habitude d'associer colère et violence. Ceci ne pouvait qu'ébranler davantage la position des théoriciens de l'agression, à qui l'idée n'était même jamais venue qu'un comportement colérique, celui d'un homme ivre par exemple, puisse varier considérablement d'une culture à l'autre. Ces recherches 
apportaient des données nouvelles, non seulement sur l'expression de la colère mais aussi sur ses véritables causes. On s'est rendu compte que, si la colère est universelle, elle se manifeste selon des règles explicites ou implicites propres à chaque culture (Tavris, 1982: 47). Par exemple, si un homme se met en colère lorsque sa femme ne remplit pas, d'après lui, les obligations liées à son rôle, c'est que dans sa culture on s'attend à ce qu'une femme remplisse de telles obligations (Ptacek, 1988). En d'autres mots, les normes culturelles nous diraient à propos de quoi nous sommes censés nous mettre en colère, et comment exprimer cette irritation. Ce qui vient compliquer les choses, c'est qu'il existe aussi des normes culturelles qui condamnent la violence familiale. Par exemple, il apparaît incorrect et lâche de frapper quelqu'un de plus faible et de plus vulnérable que soi. Cela dit, le fait de considérer l'influence culturelle ne nous aide pas vraiment à déterminer le sexe de l'agresseur car, dans une même culture, les individus sont soumis à des normes contradictoires dont les effets, nous venons de le voir, se neutralisent.

\section{Messages ambigus et conclusions douteuses}

Le cadre socio-culturel qui nous permet d'élargir notre compréhension de la violence conjugale risque, par ailleurs, d'en obscurcir les causes et de nous détourner de la question, à savoir qui est la victime et qui est l'agresseur. Bien que Gelles et Straus aient souligné comment les facteurs structurels contribuent à la violence conjugale, particulièrement dans le cadre familial, leurs abstractions, leurs généralisations et la neutralité de leurs points de référence expliquent les faiblesses de leurs conclusions. Trop dépendantes de données quantitatives, leurs études empiriques ont pris une tournure mécaniste aux applications limitées. En outre, leur condamnation absolue de la violence, où qu'elle s'exerce, a fait en sorte qu'on en est venu à considérer qu'une victime doit être, elle aussi, non violente, et ne pas riposter à la violence pour mériter qu'on l'appuie. Comme l'ont signalé Breines et Gordon (1983: 512), si nous considérons la violence comme le problème plutôt que le symptôme d'un problème, il se peut que la femme battue mérite moins de compassion si elle fait aussi usage de la force. II va sans dire que cette définition du terme "victime» est fonction de l'impuissance et de la non-violence que l'on attribue à la femme lors des altercations avec son partenaire.

De nombreux auteurs ont souligné, par exemple, que les femmes se rendent plus souvent coupables d'abus et de négligence envers les enfants, sans préciser qu'en moyenne elles sont plus nombreuses à assumer la tâche de parent. Une fois précisée l'identité du parent 
impliqué dans les cas d'enfants maltraités, il nous faut étudier le sentiment typiquement féminin de responsabilités et de culpabilité qu'éprouve une mère face à la réussite ou à l'échec dans l'éducation de son enfant. II ne faut pas non plus oublier que l'insuffisance des services de garde affecte surtout les femmes, car la garde des enfants leur incombe principalement, qu'elles vivent ou non avec un partenaire. II importe également de rappeler que, dans la majorité des cas d'abus sexuels envers des enfants, les hommes en sont les auteurs et que de façon générale, la plupart des personnes âgées victimes de violence sont des femmes et, là aussi, les hommes en sont le plus souvent les auteurs. L'accent mis sur le sexe des coupables de violence a amené Breines et Gordon à la conclusion suivante : il est remarquable que les femmes qui assument en majorité la tâche de garde des enfants ne sont impliquées que dans $50 \%$ des cas d'abus et de négligence. Cela signifie que $50 \%$ des auteurs d'abus et de négligence sont des hommes qui, en règle générale, ont rarement la responsabilité d'enfants ou encore des contacts avec eux. Cette constatation soulève une question trop souvent ignorée par de nombreux spécialistes en la matière: "Pourquoi les hommes, si peu impliqués dans la garde des enfants, maltraitent-ils tant les enfants? " (1983: 504).

À partir d'un cadre socio-culturel qui ne tient pas compte du sexe dans la dynamique de la violence conjugale, nous sommes amenés au débat lancé par Steinmentz (1978) sur le monopole de la violence, selon qui les hommes sont tout autant "victimes" de la violence conjugale que les femmes puisque des sondages ont révélé que ces dernières ont recours à la violence dans une même proportion qu'eux. Pour cette raison, Steinmentz rejette le point de vue selon lequel la violence conjugale revêt un caractère essentiellement masculin. Pourtant, ces sondages ont le défaut de ne pas faire la distinction entre un coup de pied, un coup de poing et une poussée donnés par l'un ou par l'autre car, en moyenne, la force physique des hommes est supérieure à celle des femmes. Par ailleurs, aucune question n'a été posée relativement aux blessures, aux effets, aux motifs et aux conséquences de la violence (Straus, 1979). Enfin, aucune distinction n'a été établie entre le geste d'une femme qui, en situation de légitime défense, pousse un un homme, et celui d'un homme qui, par exemple, pousse sa femme et la fait tomber dans l'escalier. On ne précise pas si la force a été utilisée en situation de légitime défense, ni pourquoi les femmes peuvent avoir fait usage d'armes dangereuses, malgré l'absence d'un danger imminent. Pourtant, ces détails sont absolument nécessaires, car dans certaines circonstances particulières, l'usage de la violence, même extrême, peut être considéré comme un comportement de légitime défense. Les études ne tiennent pas compte de la brutalité sexuelle, du harcèlement 
sexuel ou du viol, et ne mesurent pas non plus l'ampleur des outrages sociaux, affectifs et psychologiques. Cependant, toutes ces formes de violence directe et indirecte constituent la nature particulière de la violence conjugale dans notre société, chaque élément contribuant à un phénomène global qui peut être plus important que la somme de ses composantes.

Les sondages comportent aussi d'autres graves défauts qui portent atteinte à leur validité : leurs données proviennent exclusivement d'interviews effectuées auprès des hommes. Or, on sait que les agresseurs sous-estiment la violence de leurs actes, ne disent pas tout et qu'ils ont, de plus, tendance à exagérer la violence des femmes pour justifier la leur (Edleson et Brugger, 1986 ; Gondolf, 1985 ; Ptacek, 1988). Quoi qu'il en soit, ces lacunes n'ont pas découragé la publication, en Amérique du Nord, d'articles soutenant que la violence familiale est en réalité un "combat mutuel» entre l'homme et la femme, chacun pouvant être agresseur ou victime. La controverse se poursuit quant à savoir si les femmes sont aussi violentes que les hommes et si les hommes qui battent leur femme ne sont pas eux aussi des victimes (Demers, 1988 ; McNeely et Robinson-Simpson, 1987 et 1988 versus : Proud'homme, 1988 ; Saunders, 1988).

\section{Absence de contexte et concepts mal définis}

Bien que les études sociologiques aient apporté une contribution importante en dévoilant l'ampleur de la violence conjugale, on peut cependant leur reprocher de ne pas avoir tenu compte du contexte historique et social de la violence faite aux femmes, et de ne pas avoir étudié la dynamique du pouvoir et du contrôle à l'intérieur du couple. Pour découvrir la vérité en matière de violence familiale, il faut examiner d'un œil critique le contenu sémantique des termes "violence» et "victimisation" que les chercheurs utilisent couramment dans leurs travaux. En fait, étant donné que les hommes et les femmes n'ont pas la même force physique ni le même pouvoir social, on peut s'attendre à ce que les conséquences et les motifs de leur violence diffèrent aussi (Saunders, 1988).

\section{La victimisation et la condition masculine}

Bien que la perspective socio-culturelle ait permis de découvrir pourquoi la violence conjugale est une réalité collective plutôt qu'individuelle, elle n'a toutefois pas permis de trancher le débat sur la 
possibilité que les hommes soient des victimes au même titre que les femmes. Les facteurs structurels ou environnementaux, combinés aux explications psychosociales, n'ont pas fait disparaitre l'idée selon laquelle les hommes sont "victimes » des inconvénients reliées à la nécessité de se conformer aux rôles masculins traditionnels. Rien de ce que les sociologues ont fait ressortir, par exemple, n'est venu contredire l'idée que les hommes paient cher la concurrence qu'ils se font, comme le démontre l'incidence chez eux d'ulcères, de suicides, d'infarctus, de décès prématurés et de cas d'hypertension. On cite, par exemple, que les châtiments corporels de l'enfance illustrent comment on a inculqué aux hommes l'idée qu'il est légitime d'utiliser la force dans certaines circonstances, et même qu'on peut frapper quelqu'un " pour son bien " (Owens et Straus, 1974). Âl'époque où les thèmes de la « masculinité " et de la " condition masculine " prenaient racine au Québec vers la fin des années 1970 (Dulac, 1987), des responsables de groupes d'hommes ont élaboré une théorie selon laquelle les hommes oppriment les femmes parce qu'ils sont, eux-mêmes, opprimés par leur environnement. Leur violence est liée aux comportements autodestructeurs que leur impose la vie dans un monde compétitif et individualiste où ils doivent réprimer toute manifestation d'affection et d'amour afin de se conformer aux rôles masculins traditionnels.

"La dite violence est de faire subir aux autres, surtout aux femmes, le mépris de soi et nos propres tendances autodestructives. L'autodestruction prend sa source dans le fait de refouler et de nier nos insatisfactions, nos émotions, notre sensibilité par crainte de rejet, par peur de passer pour un malade mental, un hommelette ou, pire encore, pour un homosexuel ! " (Grégoire, 1980 : 122.)

Les hommes doivent se libérer de l'oppression, tout comme les femmes. L'un et l'autre sont autant victimes du sexisme et opprimés par le rapport de domination entre les sexes:

"Le sexisme pratiqué dans notre société est tel que l'homme et la femme ne peuvent entretenir de rapport égalitaire qu'en se situant de part et d'autre au cœur même de l'analyse de leur propre oppression. Ce n'est que par le rejet de leur oppression spécifique que des opprimés peuvent s'unir dans un rapport égalitaire. " (Broué, 1980 : 3334.)

Quand on donne le bénéfice du doute aux femmes sur la question de savoir qui, de l'homme ou de la femme, est le plus réprimé, il n'y a rien là que de très banal:

"On va me dire que pour les femmes, c'est pire. La situation qui leur était faite les détruisait. Je ne veux même pas contredire ce fait. Je 
pense simplement que l'important n'est pas tant de savoir ce qui est pire ou non, mais d'inventer des stratégies pour que ça change." (Chabot, 1987 : 45.)

Ou bien on a ignoré la question, ou bien on a abouti à des analyses simplistes du pouvoir détenu par les hommes, oblitérant ainsi tout débat sur les avantages réels qu'ils peuvent en retirer : "Ces apparences de pouvoir et de prestige ne nous servent-elles pas à camoufler nos sentiments de solitude et d'impuissance ? " (Grégoire, 1980 : 122). Aucun de ces théoriciens n'est parvenu à expliquer pourquoi les hommes n'ont pas réussi à se débarrasser d'une culture masculine qui fait d'eux des victimes et dont les inconvénients l'emportent sur des avantages imaginaires: "En fin de compte nous sommes les victimes du système que nous engendrons", propose Grégoire (1980:121) et Chabot (1987 : 17) en décrivant l'homme comme un individu passif et emprisonné par une aliénation spécifiquement masculine fabriquée par notre société. Pour sortir de ce dilemme, il ne suffit donc pas de renoncer au pouvoir et aux privilèges douteux qui n'ont jamais été vraiment bénéfiques :

"Changer la société patriarcale, cela veut dire [...] être en mesure de faire comprendre aux hommes que les privilèges qu'ils possèdent s'avèrent très souvent un leurre, que d'eux viennent des malaises et des maux qui provoquent plus de torts que l'on ne pensait. "(Chabot, 1987 : 44.)

Au contraire, au lieu de véritablement contester la position dominante des hommes, le changement devrait passer par "l'expression de tendresse, d'affection et d'amour [qui] est le meilleur antidote à la violence" (Grégoire, 1980 : 123). Par ailleurs, un tel changement n'est pas sans défi car "[...] les hommes ne sont dans une situation qui leur permette d'aimer librement", souligne Chabot dans son livre récent sur les hommes et l'intimité (1987: 43). Pour ces auteurs préoccupés par la condition masculine et non pas par les questions et les enjeux du pouvoir et du contrôle patriarcal, la lutte consiste par conséquent à vaincre les forces qui étouffent la pleine capacité d'un homme à aimer et à vivre plutôt que de lui faire abandonner ses soi-disant privilèges.

Il s'agit là plus que d'une tendance passagère puisque le prestigieux Journal of Family Violence vient de publier un article qui réaffirme que "les hommes violents sont aussi les victimes du processus répressif et auto-aliénant qui gouverne la rigidité inhérente aux rôles des sexes" (Jennings, 1987: 211). Auparavant, plusieurs articles sur le sujet présentaient les hommes soit comme des victimes déficientes psychologiquement et émotionnellement, soit comme des êtres confinés dans la rigidité des rôles des sexes (Purdy et Nickle, 1981; Saunders, 1984), ou 
affaiblis par la souffrance, la solitude (Trimble, 1986) et leur « impuissance » (Werk et al., 1986; Philippe, 1984 et 1986). Une revue parmi les plus importantes en la matière aux États-Unis, Social Work, a publié un article qui avance l'idée que maintenant, les hommes agresseurs sont presque « sans défense devant les gains récents du mouvement féministe " (McNeeley et Robinson-Simpson, 1987). En conséquence, les intervenants recevaient les conseils suivants : rester impartial et établir un climat thérapeutique non menaçant pour encourager la participation et instaurer la confiance chez les hommes violents (Werk et al., 1986); faire sentir à ces hommes qu'ils sont aussi des membres valables de notre société (Star, 1983) ; veiller soigneusement à ne jamais leur attribuer le blâme (Buckley, Miller et Rolfe, 1983), et à ne jamais les choquer (Currie, 1983).

Par contre, la perspective socio-culturelle permet, pour la première fois, de conceptualiser une stratégie d'intervention nouvelle et plus générale visant à mettre fin à la violence conjugale. L'objectif de l'intervention ne se limite plus simplement à amener les hommes à mettre un terme à leur comportement déviant et violent en se conformant aux normes de la société. En effet, on considère désormais que les hommes violents se conforment déjà trop à des normes indésirables qui doivent être contestées et entièrement transformées (Brod, 1987). Cette théorie permet d'introduire une notion radicale, à savoir qu'il faut changer notre société avant de pouvoir mettre fin à la violence interpersonnelle. Ainsi, ce sont toutes les instances oppressives et le système dans son ensemble qui encouragent l'inégalité et qui doivent être modifiés pour que les hommes soient en mesure de renoncer à la violence une fois pour toutes. Sur cette base, il appartient, d'une part, aux programmes destinés aux hommes violents d'intégrer la dimension sociale dans leurs séances de groupe, d'ajouter des mesures éducatives à leur répertoire d'activités (cours de formation ou campagne de publicité), et d'autre part, il faudrait exercer des pressions sociopolitiques auprès des diverses institutions sociales afin qu'un changement véritable se produise.

\section{Partage de la responsabilité et des torts}

Encore une fois, cette vision élargie de la violence conjugale n'exclut pas qu'on traite l'agresseur et la victime dans le cadre d'une même séance thérapeutique. L'objectif demeure le même, c'est-à-dire améliorer la relation conjugale (dans la mesure où elle peut être sauvée), développer au sein du couple une meilleure communication, résoudre les conflits et mettre un terme à la violence. Dans ce contexte, il n'appartient pas aux thérapeutes de faire une distinction entre clients 
violents et non violents, ni entre agresseurs et victimes. Dans les travaux portant sur les systèmes familiaux, on considère que la thérapie du couple est efficace parce qu'elle définit les problèmes sous l'angle des interactions entre les personnes et leur environnement. La violence est perçue comme un aspect d'une dysfonction continuelle à l'intérieur du couple, et l'intervention devrait donc porter sur ce trouble plutôt que sur les actes ou les choix d'un partenaire en particulier. On croit que chaque partenaire joue un rôle trop rigide à l'intérieur de la relation. C'est pourquoi on opte pour une attitude neutre et on évite de reprocher à l'homme sa conduite, de le qualifier, lui, « d'agresseur » et la femme, de "victime". Cette thérapie basée sur l'interaction a donc tendance à répartir également la responsabilité de la violence entre les deux partenaires.

\section{Pratique éclectique et méthodes d'intervention courantes}

Grâce à la combinaison d'influences psychothérapeutiques, socioculturelles et venant de l'apprentissage social, on a pu établir la base théorique permettant d'introduire des méthodes cognitivo-behavioristes et d'autres visant à réduire le stress dans le traitement des victimes et des agresseurs. On a opté pour des programmes de traitement de groupe et l'intervention a davantage été orientée vers le problème de la violence lui-même plutôt que vers la pathologie individuelle ou la relation existant au sein du couple. Le rapprochement de ces diverses perspectives a eu pour résultat l'apparition de pratiques largement éclectiques dans les programmes destinés aux hommes violents. Les intervenants impliqués s'accordent généralement pour dire qu'étant donné que la violence a pu s'apprendre dans les groupes de garçons, par exemple, elle peut aussi se désapprendre si on propose, dans un contexte de groupe, des solutions de rechange en faisant appel à diverses techniques de relaxation et de contrôle de la colère. Ainsi, les hommes apprennent de nouveaux modes d'affirmation de soi qui leur permettent de mieux contrôler leurs réactions internes et externes face au stress. On cherche également à faire en sorte que les hommes apprennent à restructurer leurs perspectives irrationnelles, dans un contexte d'entraide mutuelle. Edleson (1984), Purdy et Nickle (1981) et Saunders (1984) sont parmi les praticiens et les auteurs qui ont étudié ces possibilités. 


\section{Compromis idéologiques}

Face à cette construction théorique composée de diverses perspectives pouvant coexister dans un paradigme modifié, les responsables de programmes et les intervenants ont dû faire des choix. Par exemple, les interprétations psychologiques de la violence conjugale qui ne portent pas atteinte aux intérêts du statu quo, seraient acceptables en dépit des découvertes sociologiques établissant un lien entre la violence interpersonnelle et les institutions sociales. Il est cependant évident qu'on a tout avantage à choisir une démarche qui ne risque pas de porter atteinte aux principales sources de financement ou d'aller à l'encontre de la sagesse et des traditions de la profession. Il va sans dire, en effet, qu'on choisira de préférence les options qui entretiendront des relations cordiales avec ceux qui distribuent les ressources et réglementent les statuts et les privilèges dans notre société. Par ailleurs, en ne s'attardant pas à des considérations d'injustice, d'inégalité et d'exploitation comme facteurs de violence conjugale, on garde plus de temps pour des activités moins menaçantes et plus familières comme la rédaction de rapports, l'animation de séances thérapeutiques ou la promotion de son propre organisme. Même à l'intérieur d'une séance donnée, un choix s'impose à l'intervenant aux prises avec une clientèle violente. Il y a toujours une préférence pour l'approche thérapeutique qui, en soi, ne conteste pas trop les choses et n'exige pas une prise de position face aux questions de responsabilité dans la violence. II est beaucoup plus facile pour les praticiens d'axer leur intervention sur les émotions des participants ou sur leurs problèmes de communication, que de les confronter continuellement à leur réticence à accorder plus de pouvoir et de droits à leur partenaire.

Toutefois, un intervenant qui n'est pas constamment aux aguets de ses comportements sexistes ou collusoires risque de rendre son intervention inutile et de ne pas assumer la responsabilité de ses actes contrôlants (Hart, 1988 ; Dankwort, 1988). C'est sans doute en l'absence d'un examen critique de leur profession, de leur statut et de leurs privilèges que Straus et ses collègues préfèrent ne pas tenir compte de l'orientation pro-masculine de nombreux systèmes d'intervention tels que la religion, la santé mentale et les services médicaux (Okun, 1986 : 95). Ce n'est pas tant la valeur politique qu'ils attachent à l'expression "femme battue » à laquelle ils préfèrent " violence entre les époux " qui constitue leur plus grave erreur, mais plutôt leur incapacité ou leur refus de reconnaître la dimension politique et les limites de leur orientation positiviste, orientation dont on a la preuve lorsqu'ils qualifient l'idéologie ou le politique de "non scientifique» (Straus, 1977, 1978; Gelles, 1981). 


\section{La perspective féministe}

\section{L'aboutissement d'un mouvement populaire}

Okun (1986: 100) a affirmé que le féminisme était l'approche théorique la plus importante en ce qui concerne la violence conjugale et qu'une majorité d'auteurs traitant de ce sujet épousent les points de vue féministes. Le fait qu'il ait fallu un tel mouvement, à grande échelle, pour faire reconnaître la violence conjugale comme un problème social très répandu et grave, constitue pour les féministes plus qu'une coïncidence. Elles soulignent que ce sont leurs associations qui ont mis sur pied les premiers refuges pour femmes battues, au début des années 1970, initiative qui allait être suivie par différentes interventions des secteurs public et privé. Ces groupes posent la question épineuse : comment se fait-il que l'histoire des femmes battues n'ait que trente ans, alors que les mauvais traitements infligés aux enfants sont reconnus depuis plus d'un siècle par les services sociaux?

\section{Clarification des concepts}

La perspective féministe de la violence conjugale insiste sur le fait que, dans notre société sexiste et patriarcale, les femmes et les enfants sont les victimes de la violence tandis que les hommes en sont les auteurs. Les mauvais traitements infligés aux enfants et aux parents ainsi que la violence entre frères et sœurs sont perçus comme des dérivés de la violence faite aux femmes. Pour éviter d'atténuer l'essence de la violence faite aux femmes, les féministes distinguent le plus souvent les agressions dont les femmes sont victimes des nombreuses autres formes de violence présentes dans notre société. Par contre, cette perspective nous a permis d'établir d'importantes similitudes entre la violence subie par la femme dans une relation interpersonnelle et celle exercée contre elle par des inconnus ou des personnes familières. La révélation la plus importante est le lien établi entre le harcèlement sexuel et le viol et le fait de battre sa femme. Ceci a permis, non seulement de distinguer clairement le sexe de l'agresseur et de la victime, mais a aussi mis en lumière les attitudes critiques et humiliantes de l'opinion publique à l'égard des femmes battues et des rescapées de viol. Cette perspective nous propose une analyse qui tient compte des prétextes dont on se sert pour rejeter les torts sur la victime: les femmes sont provocantes et séductrices, c'est pour cela qu'on s'en prend à elles et qu'elles se font violer (Dobash et Dobash, 1979; Pagelow, 1981; Schechter, 1982). 
D'autres ont utilisé un paradigme féministe pour démontrer les similitudes entre les militarisme, le patriotisme chauvin, les rapports hiérarchiques à l'intérieur de la famille et la violence faite aux femmes (Roberts, 1986), d'une part, et la relation entre les mauvais traitements infligés aux enfants et la violence des hommes, d'autre part (Rush, 1980). Contrairement aux sociologues dont on a parlé plus tôt et qui rejettent les concepts politiques ou idéologiques, ces auteurs se sont servi du féminisme pour souligner et renforcer les liens entre ces phénomènes sociaux. Leur travail est représentatif du type de recherche menée au carrefour de l'oppression des classes sociales, des races et des sexes. Dans ce paradigme, on reconnaît le rôle des facteurs de société qui renforcent la violence interpersonnelle, sans pour autant diminuer la responsabilité individuelle de l'homme. La violence conjugale est perçue comme étant construite par la société et choisie par l'individu (Schechter, 1982). Au-delà de notre socialisation des rôles sexuels (la réalité externe objective), il existe d'importants intérêts personnels (nos réalités internes subjectives) qui expliquent l'existence et la reproduction de l'inégalité entre les classes, les races et les sexes dans une société qui entretient la domination, le contrôle, les abus et la violence.

L'analyse féministe clarifie le rôle des facteurs liés aux classes sociales dans la violence conjugale et indique la direction à suivre pour une stratégie d'intervention efficace. Elle explique comment le stress de la pauvreté et les facteurs qui l'accompagnent, tels que l'isolement croissant, l'absence de soutien dans l'entourage et dans la société et le manque de ressources, jouent un rôle prépondérant dans la décision des femmes de se marier ou de ne pas mettre un terme à une relation violente. Par ailleurs, cette analyse précise également pourquoi ces mêmes facteurs ne parviennent pas à expliquer la violence conjugale dans les foyers plus aisés; en effet, le statut socio-économique ne représente qu'un aspect, parmi d'autres, des rapports de pouvoir qui existent entre les hommes et les femmes dans le système patriarcal. Autrement dit, la perspective féministe permet de démontrer pourquoi la pauvreté sociale et économique rend les femmes moins susceptibles de quitter un partenaire violent que lorsqu'elles sont plus indépendantes sur le plan financier et social. En outre, si l'on replace le soutien social dans le contexte patriarcal, il est plus facile de comprendre comment un climat sexiste peut exacerber des facteurs qui contribuent à renforcer un réseau de violence masculine plutôt qu'à réduire les comportements dominants lorsque les soutiens sociaux abondent. 


\section{La dimension indispensable}

Une telle explication permet ainsi d'éviter les généralisations susceptibles de se produire lorsqu'on sépare la stratégie d'intervention du contexte socio-politique particulier de la violence conjugale. Par exemple, Bouchard, chercheur au Laboratoire de recherche en écologie humaine et sociale de l'Université du Québec à Montréal, a écrit que les programmes de prévention et d'intervention psychosociaux sur la violence familiale ne devraient pas imposer à "l'individu appauvri » la responsabilité de résoudre les problèmes socio-économiques. Bouchard n'a pas su distinguer les différents types d'intervention, auprès des hommes et auprès des femmes, et il réfute la prévention psychosociale car elle n'élimine pas " ces risques qui déterminent en grande partie le degré de vulnérabilité engendrée par l'injustice économique " (Bouchard, 1988 : 11). Même si des conditions de pauvreté peuvent aggraver la violence envers tous les membres d'une famille, l'intervention auprès des agresseurs et auprès des victimes doit être, selon l'analyse féministe, tout à fait différente. D'une part, il faut confronter les hommes, leur faire prendre conscience des gains qu'ils retirent de la domination afin de les responsabiliser pleinement. Pour leur part, les femmes reconnaissent plus facilement leur responsabilité lorsqu'elles ripostent par la force pour leur propre protection ou celle d'autrui. Une stratégie d'intervention différente s'avère donc nécessaire puisque leur sécurité et leur capacité d'agir (c'est-à-dire leur "empowerment") doivent être privilégiées. Sans perspective féministe dans l'étude de la violence familiale, il est extrêmement difficile d'échapper aux insuffisances et aux contradictions des autres analyses. Étant donné que les méthodes d'intervention de Bouchard ne tiennent pas compte du sexe, il n'est donc pas surprenant d'apprendre que ce chercheur prévoit entreprendre une étude sur la violence familiale au Québec, en se servant de la méthodologie sociologique controversée de Straus (Demers, 1988 : 68) qui ne prend pas en considération les particularités du sexe des individus.

Pour sa part, Dutton (1985) a connu des problèmes similaires car il n'a pas fait de l'analyse féministe sa principale référence. Ce chercheur met également l'accent sur l'influence du monde du travail et du voisinage dans la violence, les abus et la négligence envers les enfants, et souligne que le chômage est associé à la violence contre les femmes. Toutefois, à la différence de Bouchard, Dutton critique la perspective sociologique car "elle ne parvient pas à expliquer les différences individuelles entre les hommes» (1985: 406). Il échafaude donc un modèle écologique qui tient compte simultanément de ce qui se passe dans le foyer concerné (microsystème), des forces présentes dans les 
systèmes sociaux plus vastes où la famille est enracinée (exosystème) et des convictions et valeurs religieuses (macrosystème) qui influencent les deux autres systèmes. Il est cependant révélateur de constater que son interprétation de la dynamique du pouvoir et de la violence dans les rapports interpersonnels découle d'une hypothèse psychologique de tendance freudienne et selon laquelle, dans le système patriarcal, les hommes acquièrent le puissant besoin de conquérir et de séduire les femmes car les mères, avec une certaine ambivalence, acceptent et rejettent leurs fils dans un acte de vengeance contre leur propre oppression par les hommes. Dutton suppose qu'une fois adultes, les hommes possèdent et abandonnent alternativement leur femme pour satisfaire ces besoins d'intimité et de distance, comme le faisaient leur mère. Le caractère possessif, la jalousie et la volonté de contrôle traduisent cette anxiété ainsi que le besoin de pouvoir qui s'y rattache. Dutton fait largement appel à la théorie du contrôle social pour expliquer comment les conditions sociales contribuent à modifier la perception de la réalité. Par conséquent, nos comportements s'expliquent par l'interprétation de la stimulation physiologique et notre définition d'une telle stimulation. Selon lui, l'homme dont le seuil de " comportement, habituellement retenu, se trouve suffisamment abaissé " par ces changements, peut exprimer une "agression incontrôlée".

Dutton, qui prend bien soin de ne pas placer le féminisme au centre de sa théorie écologique, recommande "une formation pour apprendre à identifier les émotions et améliorer les aptitudes de communication " afin de renforcer le " contrôle normatif » des comportements et reconnaître "les signaux annonciateurs d'une perte de contrôle imminente ", en améliorant nos aptitudes à identifier et à catégoriser les émotions (Dutton, 1985 : 408, 410). Dépourvue d'une orientation féministe, cette théorie ne permet pas d'élucider, d'une part, pourquoi la violence peut à la fois être provoquée par l'agresseur et non provoquée par la victime et, d'autre part, qui en est responsable. Son commentaire ambigu selon lequel l'homme doit être finalement tenu responsable de la violence alors que la femme a la responsabilité "de ne pas tomber dans un rôle de victime ", illustre bien cette confusion (Dutton, 1985 : 412). Ainsi, il attribue en partie à la femme le devoir de mettre fin à la violence conjugale.

\section{Les aspects socio-politiques et personnels : une définition complète}

Les féministes établissent un rapport entre la violence faite aux femmes dans les relations interpersonnelles ou par des inconnus et les 
barrières structurelles et institutionnelles qui empêchent l'égalité des sexes. Les questions de pouvoir et de contrôle sont étudiées dans un cadre socio-politique où l'agresseur doit être confronté à ses tentatives de dominer sa partenaire par la force physique (incluant l'agression sexuelle), l'intimidation verbale et non verbale ou la torture morale. Comparée à d'autres points de vue, une telle analyse fournit une définition plus complète de la violence, à savoir tout acte qui amène la victime à faire quelque chose contre son gré, l'empêche de faire ce qu'elle veut ou encore l'amène à avoir peur (Adams, 1988).

En outre, ce point de vue tient compte du degré de force de l'agression perpétrée par un homme ou une femme, de la fréquence et de la gravité des blessures que chacun subit, de l'orientation de la violence (par exemple, de l'homme vers la femme) et de la différence entre un geste offensif et un geste défensif (Breines et Gordon, 1983; Saunders, 1988). Les actes d'un agresseur se définissent à travers les conséquences de sa volonté de domination par opposition à ses intentions déclarées. Les considérations sexistes qui sont censées inhiber la volonté du coupable et l'empêcher d'apprendre de nouvelles attitudes se trouvent remises en question. Dans les séances de groupe, on insiste sur l'usage qui est fait de la force pour préserver des privilèges et des droits et obtenir la soumission du partenaire.

\section{Stratégie d'intervention complémentaire}

En ce qui concerne l'intervention auprès des auteurs de violence conjugale, le féminisme fournit une occasion unique d'envoyer un message complémentaire aux deux partenaires. D'une part, on informe les femmes qu'elles ne sont pas responsables de leur état de victime et que c'est leur partenaire qui doit changer. D'autre part, on dit aux hommes qu'ils ne peuvent changer que s'ils assument la responsabilité entière de la violence, c'est-à-dire lorsqu'ils réalisent que la violence conjugale n'a rien à voir avec " les luttes réciproques " ou la provocation. En outre, on avise les femmes qu'elles ne sont aucunement responsables de l'absence de leur mari aux séances de thérapie, ni de leurs progrès. Par ailleurs, alors que les hommes doivent accepter d'abandonner leur pouvoir et leurs prérogatives car la violence ne se négocie pas, les femmes reçoivent de l'aide afin de renforcer leur position de force relative et négocier l'obtention d'avantages plus nombreux. Sans une telle approche, la femme et l'homme sont l'objet de messages contradictoires de la part des intervenants, puisqu'on dit aux femmes, dans les maisons d'hébergement ou à travers les services qui leur sont destinés, que la violence conjugale origine d'un rapport inégalitaire entre les 
deux sexes, alors qu'on donne aux hommes le message qu'ils agissent avec violence à cause principalement de problèmes intra-psychiques ou par réaction à un stress interne ou externe.

\section{Répercussions sur les programmes pour hommes violents}

Les programmes proféministes pour les hommes agresseurs ont comme principaux objectifs la protection et la liberté des femmes violentées, et rejettent totalement l'idée que la partenaire a quelque responsabilité dans la violence. On laisse plutôt entendre que les hommes devraient élaborer des plans de sécurité incluant le respect des craintes de la femme, entre autres, et les limites établies de la relation. Pendant le traitement, les responsables du programme prennent contact avec la partenaire afin de lui faire connaître les possibilités de protection juridique, les services de soutien, de conseil, ainsi que ceux des maisons d'hébergement pour femmes battues. En dehors de ces informations, l'intervention auprès de la femme vise à renforcer sa perception de non-responsabilité vis-à-vis la violence de son partenaire (Adams, 1988).

Les intervenants de ces programmes prennent contact avec la conjointe et travaillent en collaboration avec des organisations de femmes afin de confirmer les allégations de l'homme concernant l'absence de violence pendant tout le traitement. Ils obtiennent également sa version des événements (pas seulement celle de l'homme), ce qui permet aux membres du groupe de confronter l'individu concerné à son comportement contrôlant, et ce, dans un processus psycho-éducatif. Les hommes ont souvent des devoirs à faire entre les séances, l'objectif étant qu'ils prennent davantage conscience des besoins et des droits de leur partenaire et contribuent à une répartition plus équilibrée des tâches domestiques, entièrement assumées, en règle générale, par les femmes.

L'attitude qui consiste, pour un homme, à nier la violence ou à réduire sa responsabilité, découle de sa réticence à abandonner des privilèges, un statut, le pouvoir et l'idéologie masculine voulant qu'il existe des limites à la conscience éthique de son propre comportement (Stoltenberg, 1987). Les raisons citées par les hommes comme facteurs contribuant à leur violence font partie d'un système idéologique qui permet de justifier une position de domination comportant des avantages. C'est le concept que "EMERGE", groupe d'intervention auprès des hommes qui s'est constitué à Boston (Massachusetts), a choisi de mettre en pratique, en adoptant la théorie d'une série de comportements contrôlants et sexistes envers les femmes, et présents dans le cadre des 
relations interpersonnelles comme dans la société, à travers les institutions, les professions et les contextes politique et juridique (Adams, 1988). Les méthodes d'intervention inspirées de cette analyse tentent surtout de briser la résistance masculine à accepter la pleine responsabilité de la violence. Pour ce faire, l'homme est mis en face de situations, prérogatives et privilèges qui sont cachés par la rationalisation et minimisés par des justifications.

Par ailleurs, les programmes proféministes rejettent l'idée que les hommes battent leur femme parce qu'ils ont " perdu le contrôle d'euxmêmes", qu'ils ont trop bu, qu'ils contrôlent mal leurs impulsions, qu'ils ont "un seuil peu élevé de tolérance à la frustration ", qu'ils ont vécu une enfance traumatisante, qu'ils connaissent des difficultés économiques, ou qu'ils sont trop stressés. La violence ne peut en aucune façon s'expliquer par la description qu'on en fait. Un homme qui use de violence envers sa partenaire est en train de renforcer son contrôle sur elle. Le fait que les hommes choisissent exactement comment et qui ils frappent, démontre qu'il s'agit d'un comportement intentionnel et conditionné (Ptacek, 1988). Ils peuvent facilement remplacer la violence physique par des formes plus subtiles : contrôle économique, isolement, dénigrement, insultes, menaces d'infidélité ou froideur sentimentale. Un des problèmes fondamentaux rencontrés par des intervenants est la tendance de ces hommes à dévaloriser la femme et à la traiter comme un objet. Chaque fois que ce comportement se produit pendant une séance de groupe, ce qui semble assez fréquent, il est activement combattu. Pour ce faire, on demande aux hommes d'établir un registre de leurs comportements de contrôle plutôt que de leurs comportements colériques comme dans les programmes qui mettent en pratique les techniques de maîtrise de la colère. Cette distinction amène les participants à se concentrer davantage sur les besoins de leur partenaire et à reconnaître que la violence est un signe de leur arrogance plutôt qu'un comportement impulsif. Les comportements contrôlants et violents de chaque nouveau participant sont inscrits sur un tableau que l'on affiche à chaque séance. Par la suite, on demande aux participants de faire un "plan de responsabilités » afin d'être plus conscients des gestes qu'ils posent et de rendre la vie de leur conjointe plus sécuritaire et moins oppressive.

Contrairement aux autres perspectives, l'approche féministe ne s'oppose pas aux poursuites judiciaires. Une attitude conciliante peut traduire une approbation des comportements contrôlants et renforce l'habitude des hommes agresseurs de trouver des excuses à leur violence ou d'en rejeter la faute sur les autres. Cette attitude, qui évite la confrontation systématique des contrevenants avec leurs actes, augmente les risques pour la femme qui espère souvent que le traitement modifiera 
le comportement de son conjoint. Le processus de rupture puis de retour à la relation abusive va se prolonger encore avant que la femme puisse rompre définitivement. Cette attitude conciliante envers les agresseurs souligne également celle des agents de relations humaines et des juges qui recherchent toujours des alternatives aux sanctions pour ne pas ébranler le statu quo. De plus, les procureurs et les juges sont tenus de respecter les rouages lourds et complexes du système judiciaire qui, en soi, répond toujours mal au problème de la violence conjugale.

Selon ceux qui soulignent les limites des programmes pour hommes violents et le manque de preuves concluantes quant à leur efficacité, une démarche conciliante donne à ces hommes des messages, encore une fois contradictoires et ambigus quant à leurs responsabilités dans la violence conjugale. Si la distinction entre victime et agresseur reste vague et si les peines infligées aux agresseurs sont allégées, la bataille menée par les organisations de femmes aura échoué et la porte sera ouverte à toutes formes de répression. Déjà nous parvient des ÉtatsUnis l'écho que les policiers arrêtent les femmes victimes de violence conjugale qui ripostent par la force, et par l'intermédiaire du système judiciaire les obligent à suivre des traitements destinés à leurs agresseurs ! Le mouvement pour femmes battues lutte depuis des années pour que la violence conjugale soit enfin considérée comme un acte criminel, de façon à assurer la sécurité et la liberté de la femme. À partir de là, on pourra créer des programmes pour les hommes violents. C'est le comble de l'ironie que d'exiger des femmes qu'elles participent à ces programmes mis au point et maintenant largement répandus grâce à la bataille qu'elles ont menée pour faire reconnaître et mettre un terme à la violence conjugale.

\section{Références bibliographiques}

ADAMS, D., "A profeminist analysis of treatment models of men who batter", dans: K. Yllo et M. BoGrad (éds), Feminist Perspectives on Wife Abuse, Beverly-Hills (Cal.), Sage Publications, 1988 : 176-199.

ADAMS, D., "The continuum of male controls over women ", document inédit et distribué par EMERGE, 280 Green Street, Cambridge, Massachusetts 02139 (É.U.).

Bandura, A., Aggression: A Social Learning Analysis, Englewood Cliffs (N.J.), Prentice-Hall, 1973.

BeAUDRY, M., Les maisons des femmes battues au Québec, Montréal, Éditions Saint-Martin, 1984. 
BREINES, W. et L. GORDON, "The new scholarship on family violence ", Signs, vol. 8, $1983: 490-531$.

BROD, H., "The case for men's studies", dans: H. BROD (éd.), The Making of Masculinities: The New Men's Studies, Boston, Allen \& Unwin, 1987.

BROWNING, J., Mettre un terme à la violence conjugale : programmes canadiens de traitement des agresseurs, Ottawa, Centre national d'information sur la violence dans la famille, Santé et bien-être social Canada, 1984.

BROUE,, J., " Le groupe : Pour hommes seulement », dans : Hervé de FontenaY (éd.), La certitude d'être mâle? Une réflexion hétérosexuelle sur la condition masculine, Montréal, Les éditions Jean Basile, 1980 : 23-36.

BOUCHARD, C., "La pauvreté: comme une courbe dangereuse", Transition, vol. 18, no 3, 1988: 9-11. Publication de l'Institut Vanier de la famille, 120, avenue Holland, bureau 300, Ottawa, Ontario K1Y 0X6.

CARRIER, M. et M. MiCHAUD, "La violence faite aux femmes en milieu conjugal : le produit d'une société sexiste, une étude québécoise", Ottawa, Secrétariat d'État, Programme pour la situation de la femme, 1982.

Carrigan, T., B. Connell et J. Lee, " Hard and heavy: Toward a new sociology of masculinity», dans: M. KaufMan (éd.), Beyond Patriarchy: Essays by Men on Pleasure, Power and Change, Toronto et New York, Oxford University Press, $1987:$ 139-192.

Снавот, M., Des Hommes et de l'intimité, Montréal, Éditions Saint-Martin, 1987.

Conrad, P. et J. SCHNeIDer, Deviance and Medicalization: From Badness to Sickness, Saint-Louis (Missouri), C.V. Mosby, 1980.

CURRIE, D., Le mari violent: une approche de l'intervention, Toronto, Clark Institute of Psychiatry, 1987.

CURrie, D., "A Toronto Model", Social Work With Groups, vol. 6, no 3, $1983: 179-188$.

DANKWORT, J., "The challenge of accountability in treating wife abusers: a critique from Quebec ", Revue canadienne de santé mentale communautaire, numéro spécial: "Wife battering in Canada: a national perspective on innovative community responses", à paraître à l'automne 1988.

DANKWORT, J., "Programmes pour les hommes batteurs : une question de responsabilité ", Intervention, 79, $1988: 73-83$.

DEMERS, D., "L Le mariage : un passeport pour la violence », Châtelaine, septembre $1988: 63-73$.

DesChner, J.P., The Hitting Habit: Anger Control for Battering Couples, New York, Free Press, 1984.

DOBASH, R.E. et R. DobaSH, Violence Against Wives : A Case Against the Patriarchy, New York, Free Press (Macmillan), 1979.

DULAC, G., "Cinq essais sur la question masculine. textes sur la masculinité \# 1 ", Montréal, 1987. (Disponible, sur demande, chez l'auteur : 6850 de la Roche, app. 304, Montréal, (Qc) H2S 2E4.)

DUTTON, D.G., "An ecologically nested theory of male violence toward intimates ", dans: Sherri CLARKSON (éd.), International Journal of Women's Studies, vol. 8, no 4, 1985 : 404-413.

EDLESON, J., "Working with men who batter », Social Work, vol. 29, 1984 : 237-242. 
EDleson, J. et M. BruGger, "Gender differences in reporting of battering incidences ", Family Relations, vol. 35, 1986 : 377-382.

Farrell, W., The Liberated Man, New York, Random House, 1974.

FaulK, M., "Men who assault their wives", dans: Maria Roy (éd.), Battered Women: A Psychological Study of Domestic Violence, New York, Van Nostrand Reinhold, 1977.

Freud, S., The Basic Writings of Sigmund Freud, New York, Random House, 1938.

GANLEY, A. et L. HARRIS, "Domestic violence : Issues in designing and implementing programs for batterers", conférence donnée au American Psychological Association, le 29 août 1978.

GeLler, J.A., "Conjoint therapy: Staff training and treatment of the abuser and the abused", dans: Maria Roy (éd.), Battered Women : A Psychological Study of Domestic Violence, New York, Van Nostrand Reinhold, 1982.

GeLLES, R., "An exchange/social control theory of intrafamily violence ", conférence donnée au First National Conference for Family Violence Research, University of New Hampshire, Durham (N.H.), juillet 1981.

Gelles, R. et M. Straus, "Determinants of violence in the family: Toward a theoretical integration ", dans : W.R. BURR, R. HILL, F.I. NYE et I.L. Reiss (éds), Contemporary Theories About the Family, volume 1, New York, Free Press, 1979.

Goldberg, H., The New Male, traduit en français sous le titre: Être homme, Montréal, Éditions du Jour, 1982.

Gondolf, E.W., Men Who Batter: An Integrated Approach to Stopping Wife Abuse, Homes Beach (Florida), Learning Publications, 1985.

GrÉGOIRE, L., "La violence : une arme rentable", dans: H. DE FONTENAY (éd.), La certitude d'être mâle? Une réflexion hétérosexuelle sur la condition masculine, Montréal, Les Éditions Jean Basile, 1980 : 113-124.

GUÈVREMONT, C., "La violence masculine : des solutions ??? ", Intervention auprès des hommes, Montréal, Fédération des C.L.S.C. du Québec, Compte rendu du colloque tenu les 19 et 20 juin 1986 à l'Université du Québec à Montréal : 69-74.

HART, B., Safety for Women: Monitoring Batterers' Programs, Harrisburg (Po.), Pennyslvania Coalition Against Domestic Violence (2505 North Front Street, Harrisburg (Pa.) 17110), 1988.

JENNINGS, J.L., "History and issues in the treatment of battering men: A case for unstructured group therapy", Journal of Family Violence, vol. 2, no 3, 1987 : 193-211.

KONNER, M., "The Aggressors", dans la section "Body and Mind" du New York Times Magazine, 14 août 1988, pp. 33-34.

LAROUCHE, G., Agir contre la violence, Montréal, Les éditions de la pleine lune, 1987.

MACKInNon, C.A., Feminism Unmodified: Discourses on Life and Law, Cambridge (Mass.) et Londres, Harvard University Press, 1987.

MACleod, L., Pour de vraies amours... Prévenir la violence conjugale, Ottawa, Conseil consultatif canadien sur la situation de la femme, 1987.

MAGill, J. et J. BeNINATI, "Le pugilat conjugal ", Hom-info, vol. 5, n 2, 1984 : 20-22. 
MARTIN, M., "Hommes abusifs: le groupe de traitement sonne l'alarme", Le Droit, 23 septembre 1988, p. 6.

MCNEELY, R.L. et G. ROBINSON-SIMPSON, "The truth about domestic violence : a falsely framed issue ", Social Work, vol. 33, n 6, $1987: 485-490$. Voir aussi la réplique de Liane V. DAvis dans le numéro suivant de Social Work, vol. 34, $n^{\circ} 1,1988$ : p. 94 ; la réponse de Daniel Saunders ainsi que la réplique des auteurs McNeely et Robinson-Simpson dans Social Work, vol. 34, no 2, 1988 : 197-183 et 184-188; et également les lettres envoyées au rédacteur citées aux pages 189-191 dans le même numéro.

Mead, S., Free the Male Man !, New York, Simon \& Schuster, 1972.

NAIfEH, S., et G. White SMith, Ces hommes qui ne communiquent pas, Montréal, Le Jour, 1987. Traduit de l'Americain: Why Can't Men Open Up?, 1984.

National Coalition Against Domestic Violence (N.C.A.D.V., P.O. Box 15127, Washington, D.C., 20003-0127, É.-U.), Liste non publiée de recommandations élaborées lors d'un forum intitulé "Working With Men Who Batter" et parrainé par Domestic Abuse Intervention Project (D.A.I.P.) de Minneapolis, EMERGE de Boston, et RAVEN de St-Louis, les 8 et 9 août 1982 à Milwaukee, Wisconsin.

Nichols, J., Men's Liberation, New York, Penguin, 1975.

NorwOOD, R., Ces femmes qui aiment trop. La radioscopie des amours excessives, Montréal, Stanké, 1986. Traduit de l'Americain: Women who Love Too Much, 1985.

Novaco, R. W., Anger Control, Lexington (Mass.), Lexington Book, 1975.

OKun, L., Woman Abuse : Facts Replacing Myths, Albany (N.Y.), State University of New York Press, 1986.

OWENS, D. et M. STRAUS, "Social structure of violence in childhood and approval of violence as an adult ", Aggressive Behavior, vol. 1, n 3, 1974: 193-211.

PAGELOW, M.D., Woman-Battering : Victims and Their Experiences, Beverly Hills (Cal.), Sage, 1981.

Philibert, J., "Groupes d'aide aux hommes violents : retrouver l'homme derrière la brute", Justice, vol. 10, n० 7, $1988: 26-29$.

PHILIPPE, R., "Pro-Gam (Projet Groupes Au Masculin) », Intervention auprès des hommes, Montréal, Fédération des C.L.S.C. du Québec, Compte rendu du colloque tenu les 19 et 20 juin 1986 à l'Université du Québec à Montréal : 65-68.

PHILIPPE, R., "Sois un homme et tais-toi ! ", Hom-info, vol. 5, n 2, 1984 : 57-58.

PICARD, A., "Accepting guilt first step for sorrowful wife beaters ", Toronto Globe and Mail, 15 août 1988, p. 1.

PLECK, J.H., The Myth of Masculinity, Cambridge (Mass.), M.I.T. Press, 1981.

PROUD'HOMmE, D., "Les hommes battus : mythe ou réalité ", Journal de Montréal, 19 septembre 1988, p. 18.

PTACEK, J., "Why do men batter their wives? ", dans : K. YLLO et M. BOGRAD (éds), Feminist Perspectives on Wife Abuse, Beverly Hills (Cal.), Sage Publications, 1988 : 133-157.

PURDY, F. et N. NICKLE, "Practice principles for working with groups of men who batter ", Social Work With Groups, vol. 4, 1981 : 111-122. 
RIEKER, P.P. et E. HILBERMAN-CARMeN, "The victim-to-patient process : The disconfirmation and transformation of abuse ", American Journal of Orthopsychiatry, vol. $56, n^{\circ} 3,1986: 360-370$.

RoBerTs, B., "Ni trêve, ni abri : la guerre faite aux femmes ", Atlantis, vol. 12, no 1, $1986: 5-25$.

RondeAu, G., M. GAUVIN et J. DANKWORT, Les programmes québécois d'aide aux conjoints violents, étude sur les programmes québécois commandée par le Ministère de la santé et des services sociaux (à paraître).

SAUNDERS, D., "Wife abuse, husband abuse, or mutual combat ?", dans : K. YLLÖ et M. Bograd (éds), Feminist Perspectives on Wife Abuse, Beverly Hill (Cal.), Sage Publications, 1988a : 90-113.

SAUNDERS, D., "Other "truths" about domestic violence : a reply to McNeely and Robinson-Simpson ", Social Work, vol. 33, n 2, 1988b : 179-183.

SAUNDERS, D., "Helping husband who batter", Social Casework, vol.65, 1984 : 347-353.

SAUNDERS, D., "Marital violence: dimensions of the problem and modes of intervention", Journal of Marriage and Family Counseling, vol. 3, $n^{\circ} 1$, 1977 : 43-55.

SCheCHTER, S., Women and Male Violence: The Visions and Struggles of the Battered Women's Movement, Boston, South End Press, 1982.

SCHULTZ, L.G., "The wife assaulter : one type observed and treated in a probation agency", Journal of Social Therapy, vol. 6, $1960: 103-111$, cité dans: Lewis OKUN, Woman Abuse, op. cit., p. 79.

ShaINESS, N., "Psychological aspects of wifebattering", dans: Maria Ror (éd.), Battered Women : A Psychological Study of Domestic Violence, New York, Van Nostrand Reinhold, 1977.

SinClaIR, D., Pour comprendre le problème des femmes battues: Guide de formation pour les conseillers et les intervenants, Toronto, Librairie du gouvernement de l'Ontario, 1986.

SNell, J., R. ROSENWALD et A. Robey, "The wifebeater's wife: A study of family interaction. " Archives of General Psychiatry, vol. II, 1964 : 107-113.

Steinmann, A. et D.J. Fox, The Male Dilemma, New York, Aronson, 1974.

StEINMETZ, S.K., "The battered husband syndrome", Victimology, vol. 2, no 3-4, 1977-78 : 499-505.

StOltenberG, J., "Battering and the will to freedom", conférence donnée au Second Northeast Conference on Counseling Male Abusers, Providence (Rhode Island), 13-14 septembre 1987. (Disponible sur demande auprès de l'organisme BROTHER TO BROTHER, 1660 Broad Street, Cranston, Rhode Island, 02905.)

StORR, A., Human Aggression, Baltimore (Maryland), Penguin, 1974, cité dans : R.E. et R. Dobash, Violence Against Wives: A Case Against the Patriarchy, op. cit., $1979: 134$.

Straus, M.A., R. Gelles et S. Steinmentz, Behind Closed Doors: Violence in the American Family, New York, Anchor Press/Doubleday, 1980.

StRAuS, M.A., "A sociological perspective on the causes of family violence", dans: Maurice Green (éd.), Violence and the Family, Boulder (Colorado), 
Westview Press for the American Association for the Advancement of Science, $1980:$ 7-31.

Straus, M.A., "Measuring conflict and violence : The conflict tactics (CT) scales", Journal of Marriage and the Family, vol. 40, no 1, 1979:75-88.

Straus, M.A., "Wife-beating: How common and why?", Victimology, vol. 2, nos 3-4, 1977-1978: 443-458.

Straus, M.A. et R. Gelles, "Societal change and change in family violence from 1975 to 1985 as revealed by two national surveys ", Journal of Marriage and the Family, vol. 48, $1986: 422-430$.

TAVRIS, C., Anger: The Misunderstood Emotion, New York, Simon \& Schuster, 1982.

Тосн, H., Violent Men : An Inquiry into the Psychology of Violence, Chicago, Aldine, 1969.

TRIMBLE, D., "Confronting responsibility: Men who batter their wives", dans: A. Gitterman et L. Shulman (éds), Mutual Aid Groups and the Life Cycle, (Reproduit par Le Centre national d'information sur la violence dans la famille, Santé et Bien-être social Canada), 1986.

WALKeR, L.E., The Battered Woman, New York, Harper \& Row, 1979.

WALKER, L.E., "Battered women and learned helplessness", Victimology, vol. 2, nos 3-4, 1977-78: 525-534.

WERK, A., J. MAGILL et D. PLACK, "Traitement conjoint des couples aux prises avec des problèmes de violence conjugale : étude de suivi ", Intervention auprès des hommes, Montréal, Fédération des C.L.S.C. du Québec, Compte rendu du colloque tenu les 19 et 20 juin 1986 à l'Université du Québec à Montréal : 53-57. 